\title{
Growth of common iliac artery aneurysms coexisting with abdominal aortic aneurysms: associated factors and potential role of intraluminal thrombus
}

\author{
Yuting Wang ${ }^{1,2 \#}$, Chengcheng Zhu ${ }^{2 \#}$, Joseph Leach ${ }^{2}$, Warren Gasper ${ }^{3}$, David Saloner ${ }^{2}$, Michael Hope ${ }^{2}$ \\ ${ }^{1}$ Department of Radiology, Sichuan Academy of Medical Sciences and Sichuan Provincial People's Hospital, University of Electronic Science \\ and Technology of China, Chengdu 610072, China; ${ }^{2}$ Department of Radiology and Biomedical Imaging, ${ }^{3}$ Department of Surgery, University of \\ California, San Francisco, CA, USA
}

"These authors contributed equally to this work.

Correspondence to: Chengcheng Zhu, PhD. 4150 Clement Street, San Francisco, CA 94112, USA. Email: Chengcheng.Zhu@ucsf.edu; Yuting Wang, MD. No. 32, Section 2, 1st Ring Road (West), Chengdu 610072, China. Email: wangyuting_330@163.com.

\begin{abstract}
Background: The factors influencing common iliac artery aneurysm (CIA) growth are not fully known. Intraluminal thrombus (ILT) has been studied as a marker of growth in abdominal aortic aneurysms (AAA), but its role in CIAs is unknown. This study aims to examine the factors associated with growth of CIAs coexistent with AAA using serial cross-sectional imaging (CT and MRI) with multiplanar reconstruction (MPR).

Methods: Patients with synchronous AAA and CIA observed at contrast-enhanced CT or MRI were included. The maximal diameters of both CIA and AAA were measured using MPR. Correlation of the baseline aneurysm diameter and growth rate between CIA and AAA was evaluated. Multivariate regression analysis was used to investigate the factors associated with CIA growth.

Results: Seventy-five AAA patients (age 74 9 years; all male) with 100 CIAs were followed for an average of $2.2 \pm 1.2$ years. CIA and AAA growth were positively correlated $(\mathrm{r}=0.39, \mathrm{P}<0.001)$. Multivariate analysis showed that CIA baseline diameter, AAA baseline diameter, and smoking were positively related to CIA growth. In 2-3 cm CIAs ( $\mathrm{n}=59)$, ILT tends to be an independent predictor of AAA growth $(\mathrm{P}=0.076)$, and CIAs with ILT grow at more than twice the rate of CIAs without ILT (1.7 vs. $0.8 \mathrm{~mm} / \mathrm{year}, \mathrm{P}=0.036)$, despite similar baseline diameters.

Conclusions: CIA baseline diameter, coexisting AAA baseline diameter, and smoking are associated with CIA growth. In CIAs measuring $2-3 \mathrm{~cm}$, the presence of ILT is associated with faster growth, and should be taken into account when determining surveillance intervals and timing of intervention for patients being considered for AAA repair.
\end{abstract}

Keywords: Iliac artery aneurysm; abdominal aortic aneurysm (AAA); thrombosis; computed tomography (CT)

Submitted Oct 21, 2019. Accepted for publication Feb 07, 2020.

doi: $10.21037 /$ qims.2020.02.12

View this article at: http://dx.doi.org/10.21037/qims.2020.02.12

\section{Introduction}

Common iliac aneurysms (CIAs) are frequently identified during routine abdominal aortic aneurysm (AAA) screening and surveillance (1). Up to half of patients with an AAAs have an iliac artery aneurysm, and of these $70 \%$ are CIAs (2-4).
Although typically asymptomatic $(3,5)$, CIAs expand over time with a potential for life-threatening rupture, similar to that of AAAs (6). CIAs also complicate the management of AAA disease: iliac artery aneurysms can rupture after AAA repair, and following endovascular AAA repair, patients 
with concomitant iliac artery aneurysms have higher rates of distal stent-graft endoleak and iliac limb occlusion $(7,8)$. Despite their clinical importance, clinical follow-up data on CIAs is scarce.

Risk factors for AAA growth are well studied, with literature supporting or proposing the use of baseline aneurysm diameter, intraluminal thrombus (ILT) burden and composition, high mechanical wall stress, smoking status, and other clinical factors to guide surveillance strategies (9-12). However, the few studies of CIA disease have focused mainly on aneurysm diameter, which is correlated with symptomatic disease and rupture risk $(3,13)$. Given the linkage between the two disease processes, we sought to determine if other AAA risk factors may be predictive of progressive CIA disease. In particular, while ILT burden has been proposed as a marker of faster AAA growth $(10,14)$, the impact of ILT on CIA growth has not been studied.

This study aims to identify the factors associated with CIA growth in patients with coexistent AAA disease, and examine the role of ILT in CIA progression. Previous studies measuring CIA growth relied predominantly on ultrasound, the accuracy and reproducibility of which is limited in many common clinical scenarios $(4,15)$. Most prior cross-sectional imaging studies do not employ standardized multiplanar reconstruction (MPR) to measure CIA diameter, or had relatively small sample sizes and short follow-up duration (10). To address these shortcomings, we intend to use serial high-resolution cross-sectional imaging (CT or volumetric black blood MRI) with standardized MPR in a large cohort of patients with CIA disease.

\section{Methods}

\section{Study population}

The institutional review board of the San Francisco Veterans Affairs Health Care System (SFVAHCS) approved this retrospective study; the requirement for patients' written consent was waived. Patients who underwent contrast-enhanced CT or high-resolution black blood MRI of the abdomen and pelvis were selected using radiology records from February 2000 to April 2018 if one of their exams demonstrated a AAA and a subsequent cross-sectional imaging study was available for follow-up comparison. Studies were further screened to identify AAA patients with coexisting CIA disease. We defined a CIA as a dilated common iliac artery with a diameter of greater than $1.8 \mathrm{~cm}$ $(3,6,13,16)$. The inclusion criteria for our study were: (I) the presence of AAA (abdominal aorta diameter of $3 \mathrm{~cm}$ or greater) and coexisting CIA on a baseline contrast enhanced CT or MRI; and (II) availability of at least one follow-up CT or MRI between 6 months and 5 years after the baseline exam. The exclusion criteria for our study were: (I) open or endovascular repair of a CIA; and (II) poor image quality precluding reliable aneurysm measurement. Demographic and clinical information of the study cohort, including age, sex, smoking history, and coexistence of hypertension, diabetes, and ischemic heart disease were also recorded.

\section{Image acquisition}

All CT studies were acquired helically on clinical CT scanners, using standard institutional protocols. The initial exam for each patient was a contrast enhanced study and a range of CT techniques are represented in the data, as no strict requirement was made about the specific imaging protocol applied. Protocols included CT angiography, routine portal venous phase $\mathrm{CT}$, multiphase imaging for liver or pancreatic mass evaluation, and CT Urography. The overwhelming majority ( $90 \%$ ) of exams were performed on GE scanners, including Lightspeed 16, Lightspeed Ultra, Lightspeed VCT, Discovery CT750 HD, and Revolution CT. $120-\mathrm{kVp}$ tube potential was common to all studies with the exception of those performed on a dual energy system, and automatic tube current modulation was applied on all exams. A variety of bolus timing and tracking strategies were applied across the different protocols and years in which exams were performed. Images were reconstructed at 1- to 5 -mm slice thickness.

Black blood MRI exams were acquired on a $3 \mathrm{~T}$ wholebody system (MAGNETOM Skyra, Siemens Healthcare, Erlangen, Germany) with an 18-channel body coil. A fastspin-echo sequence using variable flip angle (SPACE) with DANTE blood suppression was used (DANTE-SPACE) $(17,18)$. Images were acquired in the coronal plane during free breathing, covering the abdominal aorta from the renal arteries to the proximal iliac arteries. Imaging parameters: $\mathrm{TR} / \mathrm{TE}=800 / 20 \mathrm{~ms} ; 32 \times 32 \mathrm{~cm}^{2}$ field of view (FOV); 52 coronal slices with $1.3 \mathrm{~mm}$ slice thickness. $1.3 \mathrm{~mm}$ isotropic resolution, and scan time of 7 minutes.

\section{Image analysis}

All images were transferred to an offline workstation, and analysis was performed with commercial image viewing and processing software (Horos, 3.3.5). Two reviewers with 6 

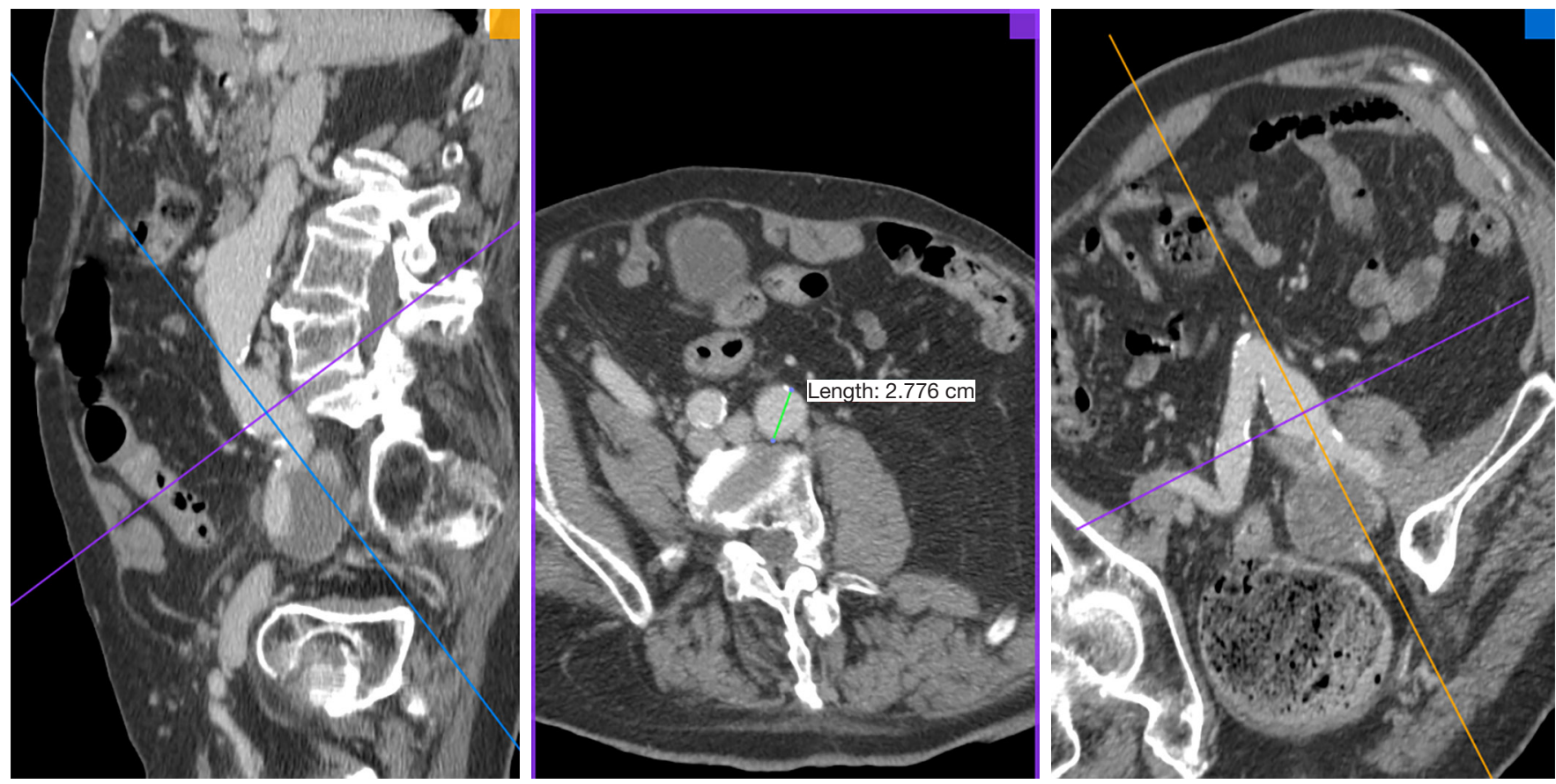

Figure $1 \mathrm{MPR}$ on CT to measure the maximum diameter of CIA. Noted that the location of iliac artery is deep and the geometry is tortuous.

and 7 years' experience in reviewing abdominopelvic CT and MR images performed the image review and aneurysm measurements. The maximal diameters of the CIAs were measured orthogonal to the long axis of the vessel using the MPR method (18) (Figure 1). The CIA and coexisting AAA diameters at the earliest time point (baseline diameters) and the latest available time point (follow-up diameters) were measured independently, and the aneurysm growth rate ( $\mathrm{mm} /$ year) was calculated as (follow-up diameter -baseline diameter)/follow-up duration (years). At baseline, ILT was defined as present if the ILT plus vessel wall thickness was greater than $5 \mathrm{~mm}$. When it was difficult to differentiate ILT from intramural hematoma in a few cases, it was not considered as ILT if the signs of calcification ingression or penetrating atherosclerotic ulcer were observed. The presence of ILT in CIA and coexisting AAA was recorded. At the maximal diameter location of each CIA, the maximal ILT thickness was recorded. In 40 randomly selected datasets, both reviewers measured the CIA diameter separately and the inter-observer agreement was evaluated.

\section{Statistical analysis}

Data were assessed for normality by using the Shapiro-
Wilk test. Continuous data were summarized using the mean \pm standard deviation. Categorical data were expressed as counts or percentages. Continuous data were compared using either a Mann-Whitney U test or Student's $t$-test. Categorical variables were analyzed using the Chi Square test. Spearman's $r$ was used to describe the correlation between measured parameters. The reproducibility of measurements between two observers was evaluated by intraclass correlation coefficient (ICC). Measurement error between scans was quantified by the coefficient of variance (CV, defined as standard deviation between measurements/ mean $\times 100 \%)$. Power analysis based on a two-sample unpaired $t$-test (two-sided) was performed with $90 \%$ power and $5 \%$ significance level to denote statistical significance $(19,20)$. Since some patients had bilateral CIAs, the growth rate of which might be correlated with each other, statistical adjustment for this factor was made. Linear mixedeffects models were therefore employed for univariate and multivariate analysis to identify the independent predictors of aneurysm growth. A P value of less than 0.05 was considered statistically significant. All $\mathrm{P}$ values were two-sided. SPSS (Statistics Version 26) was used for data analysis. 
Table 1 Patient demographics

\begin{tabular}{lc}
\hline Patient demographics & $\mathrm{N}=75$ \\
\hline Age, mean \pm SD & $74.0 \pm 8.5$ \\
Male & $75(100 \%)$ \\
BMI $\left(\mathrm{kg} / \mathrm{m}^{2}\right)$, mean $\pm \mathrm{SD}$ & $27.4 \pm 6.0$ \\
Hypertension* & $53(70.7 \%)$ \\
DM & $20(26.7 \%)$ \\
Smoking & $19(25.3 \%)$ \\
\hline
\end{tabular}

*, hypertension is defined as resting blood pressure $>140 / 90$ $\mathrm{mmHg}$. BMI, body mass index; DM, diabetes mellitus.

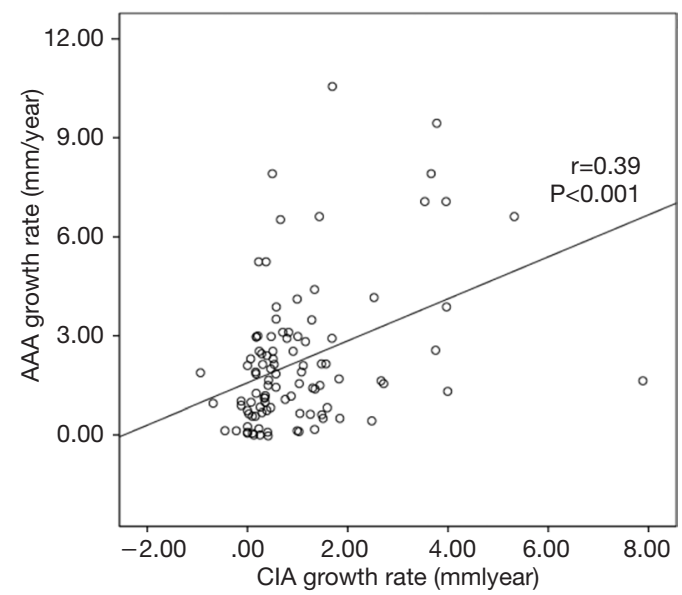

Figure 2 The correlation of the growth rate of CIA and AAA.

\section{Results}

Between 2000 and 2018, 230 patients with AAA were imaged more than once with CT and/or MRI. Among them, 81 patients with 107 CIAs were identified. One patient with a unilateral CIA was excluded for follow-up less than 6 months, and 2 patients (with a total of 3 CIAs) were excluded for follow-up time longer than 5 years. Three patients, each with unilateral CIAs, were excluded due to unsatisfactory image quality. Our final study cohort was comprised of 75 patients with 100 CIAs that were followed up for an average of $2.2 \pm 1.2$ years; patient demographics are shown in Table 1. Twenty-seven patients (36.0\%) had bilateral CIAs. CIAs were more commonly observed on the right (55.0\%). All 75 patients underwent contrast-enhanced $\mathrm{CT}$ as the baseline exam. Nine patients had volumetric black blood MRI at the latest follow up assessment, while the remaining patients were imaged by CT for the latest follow up.

\section{CIA growth rate}

CIAs had an overall baseline diameter of $2.4 \pm 0.6 \mathrm{~cm}$ with growth rate of $1.0 \pm 1.3 \mathrm{~mm} /$ year. There were no significant differences in baseline diameter or growth rate between left and right CIAs ( $\mathrm{P}=0.862,0.671$ respectively), or between bilateral and unilateral CIAs $(\mathrm{P}=0.386,0.378$ respectively).

\section{Correlation between $C I A$ and coexisting $A A A$}

CIA growth was positively correlated with AAA growth $(r=0.39, \mathrm{P}<0.001)$ (Figures 2,3), whereas the baseline diameters of CIA and AAA were not significantly correlated $(\mathrm{P}=0.583)$. Twenty-seven CIAs $(27.0 \%)$ contained ILT, with the coexisting AAA containing ILT in 21/27 instances. In the remaining 73 CIAs without ILT, ILT within the coexisting AAA was seen only 35 times. The presence of ILT in a CIA was associated with that in the coexisting AAA $(\mathrm{P}<0.01)$.

\section{Univariate and multivariate analysis to identify factors associated with CIA growth, and the potential role of ILT}

Univariate and multivariate analysis showed that CIA baseline diameter, baseline diameter of coexisting AAA, and smoking were independent risk factors for CIA growth, as shown in Table 2. Diabetes tended to have a protective effect from CIA growth but this did not reach significance.

There were significant differences in baseline diameter and growth rate between CIAs with $(\mathrm{n}=27)$ and without $(\mathrm{n}=73)$ ILT. Specifically, in CIAs measuring $2-3 \mathrm{~cm}(\mathrm{n}=59)$, which represented the largest subgroup (59.0\%), the growth rate of CIA with ILT was more than double that of CIAs without ILT (1.7 vs. $0.8 \mathrm{~mm} /$ year, $\mathrm{P}=0.036$ ), whereas the baseline CIA diameter did not differ significantly between groups with and without ILT (Table 3, Figures 4,5). In CIAs with ILT ( $\mathrm{n}=27)$, ILT thickness was significantly correlated with aneurysm growth rate $(\mathrm{r}=0.50, \mathrm{P}=0.007)$. Among the parameters identified as risk factors in the whole group, only ILT tended to be predictive of CIA growth rate in the $2-3 \mathrm{~cm}$ subgroup, with a borderline $\mathrm{P}$ value of 0.076 (Table 4). 

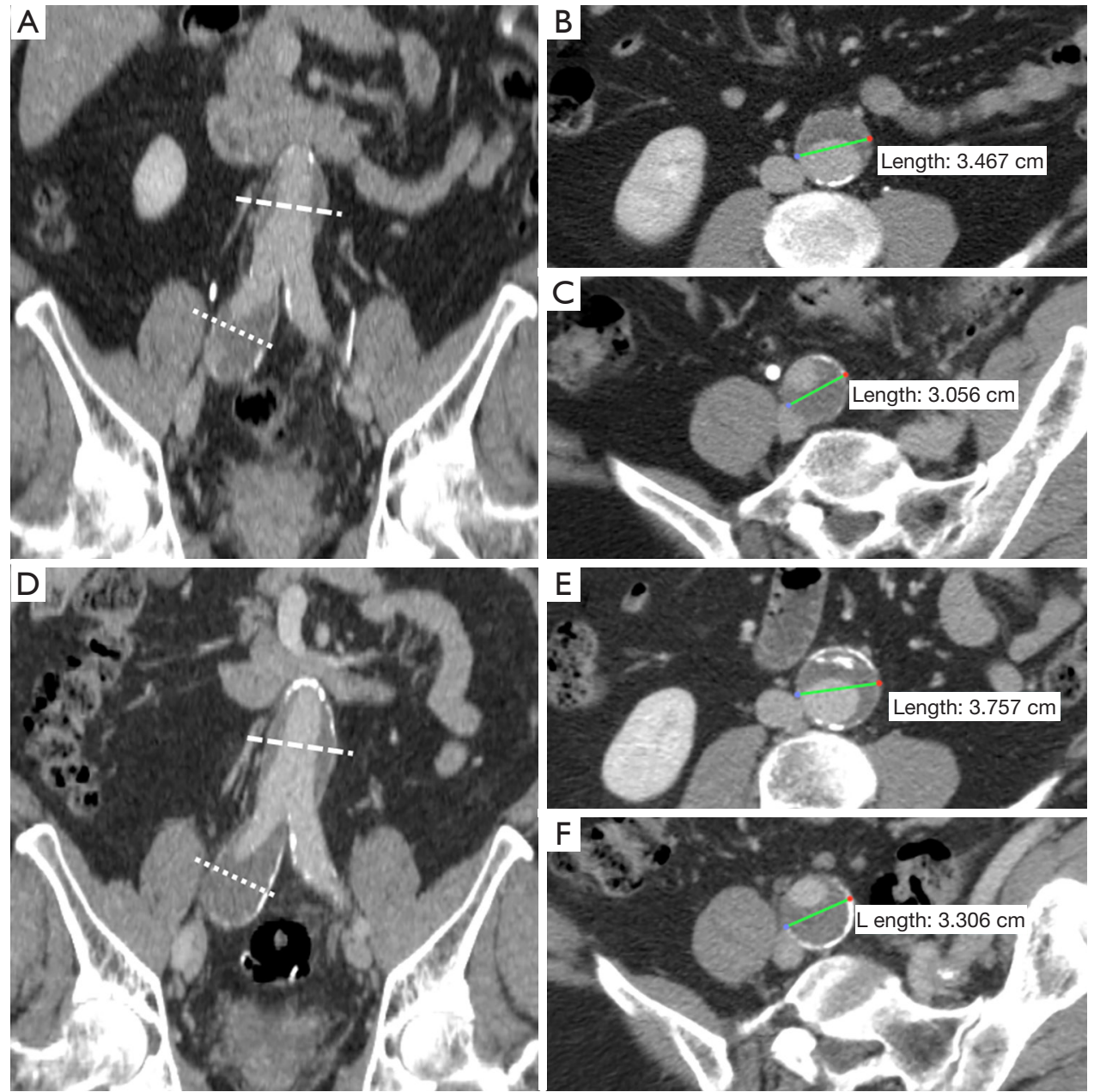

Figure 3 A 72 years old male with a right CIA with ILT, along with a AAA with ILT. CT shows detailed aorto-iliac anatomy, and both aneurysms grow after 3.1 years of surveillance. (A) MPR of the baseline CT images; (B) measurement of the AAA at baseline; (C) measurement of the right CIA at baseline; (D) MPR of the follow-up CT images; (E) measurement of the AAA on the follow-up study; (F) measurement of the right CIA on the follow-up study.

Table 2 Uni- and multi-variate analysis by linear mixed effect model for CIA growth rate

\begin{tabular}{|c|c|c|c|c|c|c|}
\hline \multirow{2}{*}{$\mathrm{CIA}$ growth rate } & \multicolumn{3}{|c|}{ Univariate analysis } & \multicolumn{3}{|c|}{ Multivariate analysis } \\
\hline & Estimate & SD & $P$ & Estimate & SD & $\mathrm{P}$ \\
\hline Baseline diameter & 0.074 & 0.022 & $0.002^{*}$ & 0.067 & 0.023 & $0.005^{\star}$ \\
\hline ILT & 0.084 & 0.030 & $0.006^{*}$ & 0.026 & 0.032 & 0.423 \\
\hline AAA baseline diameter & 0.039 & 0.015 & $0.012^{*}$ & 0.042 & 0.012 & $0.001^{*}$ \\
\hline $\mathrm{BMI}$ & 0.000 & 0.002 & 0.940 & NA & - & - \\
\hline Smoking & 0.091 & 0.031 & $0.004^{*}$ & 0.064 & 0.027 & $0.021^{\star}$ \\
\hline DM & -0.046 & 0.032 & 0.148 & NA & - & - \\
\hline HTN & 0.052 & 0.031 & 0.101 & NA & - & - \\
\hline
\end{tabular}

${ }^{*}, \mathrm{P}<0.05 . \mathrm{NA}$, not applicable. 
Table 3 Growth rate of CIAs by diameter range and ILT status

\begin{tabular}{|c|c|c|c|c|c|}
\hline $\begin{array}{l}\text { Subgroup based on } \\
\text { baseline diameter }(\mathrm{N})\end{array}$ & ILT & $\begin{array}{l}\text { Baseline diameter } \\
(\mathrm{cm}), \text { mean } \pm S D\end{array}$ & $\mathrm{P}$ & $\begin{array}{l}\text { Growth rate }(\mathrm{mm} / \\
\text { year), mean } \pm S D\end{array}$ & $\mathrm{P}$ \\
\hline All $(n=100)$ & Y: 27 (27.0\%) & $2.9 \pm 0.7$ & $<0.001^{*}$ & $1.6 \pm 1.8$ & $0.003^{\star}$ \\
\hline$<2 \mathrm{~cm}(\mathrm{n}=27)$ & Y: 2 (7.4\%) & $2.0 \pm 0.1$ & 0.088 & $0.5 \pm 0.0$ & 0.783 \\
\hline $2-3 \mathrm{~cm}(\mathrm{n}=59)$ & $\mathrm{N}: 47$ & $2.3 \pm 0.3$ & & $0.8 \pm 1.0$ & \\
\hline \multirow[t]{2}{*}{$>3 \mathrm{~cm}(\mathrm{n}=14)$} & Y: 13 (92.9\%) & $3.6 \pm 0.4$ & 0.539 & $1.8 \pm 1.5$ & 0.175 \\
\hline & $\mathrm{N}: 1$ & 3.9 & & 4.0 & \\
\hline
\end{tabular}

${ }^{*}, \mathrm{P}<0.05 . \mathrm{Y}$, with ILT; N, without ILT.

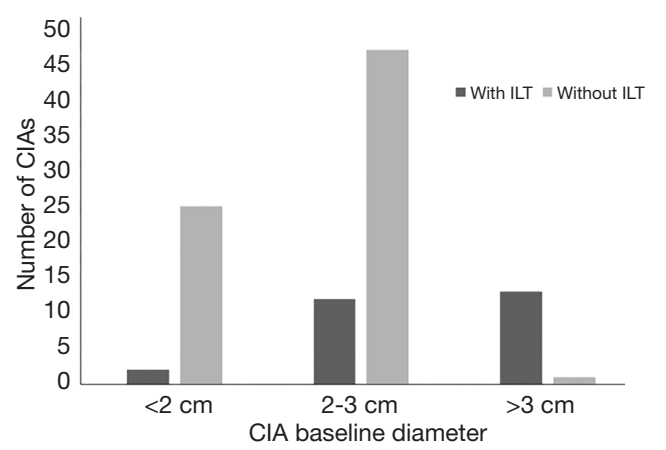

Figure 4 The ILT prevalence in three subgroups of CIAs based on their baseline diameters.
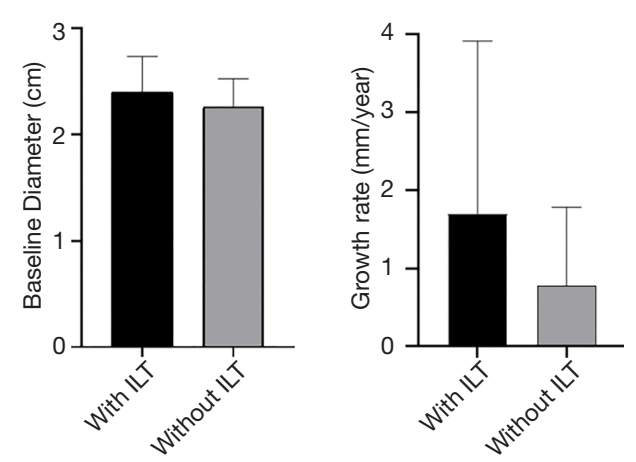

Figure 5 Comparison of CIA baseline diameter and growth rate between subgroups with and without ILT in CIAs of $2-3 \mathrm{~cm}$.

\section{Reproducibility of imaging measurement and power analysis}

There was an excellent interobserver agreement for CIA diameter measurement, with ICC of 0.993 (95\% CI, 0.987-
0.996) and CV of 3.9\%, without significant bias $(\mathrm{P}=0.99)$.

Power analysis based on the measurement error of aneurysm diameter change (SD of the difference of aneurysm diameter change measured by two observers $=0.98 \mathrm{~mm}$ ) was performed. In CIAs with diameters of $2-3 \mathrm{~cm}, 5$ patients in each group (assuming two equal sample groups) were required in order to detect a $90 \%$ difference [as (1.7-0.8 mm/year) $/ 1.0 \mathrm{~mm} /$ year) $=90 \%$ ] in aneurysm growth rate between CIA with and without ILT. In our study, sample groups of CIAs with and without ILT were unequal (12 vs. 47), and the adjusted required sample sizes were 3 and 12 for CIA with and without ILT, respectively. The above analysis illustrates that our sample sizes are sufficient for statistical power. Furthermore, for two equal sample groups, 104, 17, or 6 patients would be required in each group to detect 20\%, $50 \%$, and $80 \%$ differences of CIA growth rate, respectively.

\section{Discussion}

In this study, predictive factors and the role of ILT in CIA growth were explored using serial cross-sectional imaging with MPR measurements performed. While ILT has been studied in the context of AAA disease (14), this is the first study to investigate the role of ILT in CIAs. We observed differences in baseline diameter and growth rate between CIAs with and without ILT, as well as a significant correlation between ILT thickness and growth rate in CIAs containing ILT. In CIAs measuring 2-3 cm, the presence of ILT was associated with more than 2-fold faster growth.

It is commonly recommended that a $3 \mathrm{~cm}$ CIA be 
Table 4 Univariate analysis by linear mixed effect model for CIA growth rate in 2-3 cm subgroup

\begin{tabular}{lccc}
\hline \multirow{2}{*}{ CIA growth rate } & \multicolumn{3}{c}{ Univariate analysis } \\
\cline { 2 - 4 } Baseline diameter & Estimate & SD & 0.197 \\
ILT & 0.073 & 0.055 & 0.076 \\
AAA baseline diameter & 0.082 & 0.044 & 0.243 \\
Smoking & 0.028 & 0.024 & 0.136 \\
\hline
\end{tabular}

considered for repair due to an increased risk of symptoms and aneurysm rupture (21-23). In our study, CIAs measuring 2-3 cm comprised the largest subgroup by size, and a subset for which surveillance intervals should be carefully tailored given the possibility of near-term intervention. Our results suggest that the presence of ILT may be an important risk factor for progressive disease in this subgroup: the growth rate was more than double in CIAs with ILT compared to those without, despite comparable baseline aneurysm diameters. While the presence of ILT was not identified overall as an independent risk factor of CIA growth in multivariate regression analysis, this may be explained by the fact that 13 of 14 large $(>3 \mathrm{~cm})$ CIAs contained ILT, and their growth rate may be predominantly affected by their already large size, as is the case in AAA. ILT, however, tended to be predictive of CIA growth rate in the $2-3 \mathrm{~cm}$ subgroup $(\mathrm{P}=0.076)$.

The underlying mechanisms by which ILT may influence aneurysm growth are not fully understood. In the AAA literature, an earlier study indicated a positive correlation between thrombus area fraction and AAA expansion rate (24). A more recent study, however, reported a negative correlation between the relative size of ILT and aneurysm expansion rate, suggesting that the growth of AAAs was slowed by thicker thrombus (25). These conflicting results highlight two likely coexistent and contrasting influences of thrombus on aneurysm evolution: (I) a mechanical theory posits that ILT acts as a "cushion" or protective layer that reduces the mechanical vessel wall stress $(24,26)$; whereas (II) an alternate theory argues that the hypoxia and enzymatic/ inflammatory processes induced by ILT could weaken the underlying aneurysm wall (27-29). These two opposing factors likely exist along a spectrum. This is seen in the complexity and heterogeneity of ILT composition, with the fresher luminal layer being more enzymatically active than older thrombus layers. Whereas CT has limited ability to reveal the heterogeneity of ILT, a recent study using black blood MRI to assess the different signal intensity/features of ILT in AAA revealed that AAAs with T1 hyperintense ILT are larger in diameter and grow faster (12). ILT composition and heterogeneity warrant further investigation.

Our study finds an average CIA growth rate of $1.0 \mathrm{~mm} /$ year, similar to previous studies by Santilli et al. and Richards et al. $(4,6)$, and lower than that found by Huang et al., possibly due to their employment of mixed assessment modalities (3). Identified independent risk factors for the whole CIA group by multivariate regression included CIA baseline diameter, coexisting AAA baseline diameter, and smoking. Results showed a considerable association between CIA growth and growth of a coexisting AAA, suggesting that both aneurysms might share systematic vascular pathogenic factors, and that surveillance of coexisting CIA is necessary during AAA surveillance. Diabetes tended to be protective against CIA growth as reported for AAA, but the effect did not reach statistical significance (10).

Previous studies have typically employed ultrasound or mixed modality assessment and measurement of the anteroposterior diameter of abdominal aortic and iliac artery aneurysms. However, the iliac arteries are located deep within the body, with often markedly tortuous geometries as shown in Figure 1, making measurement by ultrasound highly operator-dependent and not ideal for the assessment of slow-growing CIAs $(3,4,6)$. A meta-analysis revealed that the repeatability coefficients of anteroposterior aortic diameter measurements was nearly $5 \mathrm{~mm}$ (range, $1.6-10.5 \mathrm{~mm}$ ) by ultrasound (30), which is approximately 5 times larger on average than that in our study employing high-resolution cross-sectional imaging and MPR analysis (repeatability coefficient: $0.98 \mathrm{~mm}$ ). Therefore, our study has greater statistical power than previous studies due to a more reliable measurement technique.

One unique issue in the evaluation of CIA disease is that some patients may have bilateral disease with correlated growth rates. Our study employed a linear mixed effects 
model for statistical correction for this factor, generating more reliable statistic results. Another strength of the present study is the provision of a power calculation to validate the statistical effects of the current analysis, with further sample sizes presented to aid future studies aimed at examining differences in aneurysm expansion rates between two groups.

There are several limitations of our study. First, only male patients were included because of the institutional practice conditions in the VA healthcare system. Nevertheless, we note that in the general population it is reported that $81 \%$ to $100 \%$ of CIA patients are men, and men constituted more than $80 \%$ of subjects in prior studies $(3,4,10)$. Second, this is a single-center retrospective study, and although inclusion and exclusion criteria are not overly complex, selection bias cannot be excluded. Third, the modalities used for aneurysm surveillance were not uniform, since 9 patients had volumetric black blood MRI as their latest follow up exam. However, previous studies showed that black blood MRI is as accurate as CT and the vessel measurements should be interchangeable (18). Fourth, although the comparison of the most recent study and the baseline study is widely used in practice to assess the expansion rate of an aneurysm, an episodic expansion might occur and cannot be fully reflected in the average expansion rate. Future prospective studies with regular short follow-up intervals for CIAs are suggested to better address this issue with greater reliability. In addition, although this study used maximal diameter on MPR to evaluate CIA and maximal thickness to quantify ILT, more sophisticated segmentation and geometric analysis methods can be explored in the future to calculate the volumes and more sophisticated descriptors of CIAs and their ILT.

\section{Conclusions}

In conclusion, baseline CIA diameter, baseline diameter of coexisting AAA, and smoking are associated with CIA growth. In CIAs measuring 2-3 cm, presence of ILT is associated with faster aneurysm growth, and should be considered when determining surveillance intervals and timing of intervention for patients being considered for AAA repair.

\section{Acknowledgments}

Funding: The scientific guarantor of this publication is Michael D. Hope. This study has received funding by
United States National Institutes of Health (NIH) grants R01HL114118 and R01HL123759. The authors thank Dr Yingjie Li for helping refine the methodology.

\section{Footnote}

Conflicts of Interest: The authors have no conflicts of interest to declare.

Ethical Statement: The institutional review board of the San Francisco Veterans Affairs Health Care System (SFVAHCS) approved this retrospective study; the requirement for patients' written consent was waived.

Open Access Statement: This is an Open Access article distributed in accordance with the Creative Commons Attribution-NonCommercial-NoDerivs 4.0 International License (CC BY-NC-ND 4.0), which permits the noncommercial replication and distribution of the article with the strict proviso that no changes or edits are made and the original work is properly cited (including links to both the formal publication through the relevant DOI and the license). See: https://creativecommons.org/licenses/by-nc-nd/4.0/.

\section{References}

1. Ashton HA, Buxton MJ, Day NE, Kim LG, Marteau TM, Scott RA, Thompson SG, Walker NM, Multicentre Aneurysm Screening Study G. The Multicentre Aneurysm Screening Study (MASS) into the effect of abdominal aortic aneurysm screening on mortality in men: a randomised controlled trial. Lancet 2002;360:1531-9.

2. Krupski WC, Selzman CH, Floridia R, Strecker PK, Nehler MR, Whitehill TA. Contemporary management of isolated iliac aneurysms. J Vasc Surg 1998;28:1-11; discussion 11-3.

3. Huang Y, Gloviczki P, Duncan AA, Kalra M, Hoskin TL, Oderich GS, McKusick MA, Bower TC. Common iliac artery aneurysm: expansion rate and results of open surgical and endovascular repair. J Vasc Surg 2008;47:1203-10; discussion 1210-1.

4. Richards T, Dharmadasa A, Davies R, Murphy M, Perera $\mathrm{R}$, Walton J. Natural history of the common iliac artery in the presence of an abdominal aortic aneurysm. J Vasc Surg 2009;49:881-5.

5. Melas N, Saratzis A, Dixon H, Saratzis N, Lazaridis J, Perdikides T, Kiskinis D. Isolated common iliac artery aneurysms: a revised classification to assist endovascular 
repair. J Endovasc Ther 2011;18:697-715.

6. Santilli SM, Wernsing SE, Lee ES. Expansion rates and outcomes for iliac artery aneurysms. J Vasc Surg 2000;31:114-21.

7. Hobo R, Sybrandy JE, Harris PL, Buth J, Collaborators E. Endovascular repair of abdominal aortic aneurysms with concomitant common iliac artery aneurysm: outcome analysis of the EUROSTAR Experience. J Endovasc Ther 2008;15:12-22.

8. Mylankal KJ, Baxter Z, Perry EP. Common iliac aneurysm rupture after previous aortic aneurysm resection. Ann Vasc Dis 2010;3:74-6.

9. Sweeting MJ, Thompson SG, Brown LC, Powell JT, collaborators R. Meta-analysis of individual patient data to examine factors affecting growth and rupture of small abdominal aortic aneurysms. Br J Surg 2012;99:655-65.

10. Hendy K, Gunnarson R, Golledge J. Growth rates of small abdominal aortic aneurysms assessed by computerised tomography--a systematic literature review. Atherosclerosis 2014;235:182-8.

11. Chaikof EL, Dalman RL, Eskandari MK, Jackson BM, Lee WA, Mansour MA, Mastracci TM, Mell M, Murad MH, Nguyen LL, Oderich GS, Patel MS, Schermerhorn ML, Starnes BW. The Society for Vascular Surgery practice guidelines on the care of patients with an abdominal aortic aneurysm. J Vasc Surg 2018;67:2-77.e2.

12. Zhu C, Leach JR, Tian B, Cao L, Wen Z, Wang Y, Liu X, Liu Q, Lu J, Saloner D, Hope MD. Evaluation of the distribution and progression of intraluminal thrombus in abdominal aortic aneurysms using high-resolution MRI. J Magn Reson Imaging 2019;50:994-1001.

13. Bacharach JM, Slovut DP. State of the art: management of iliac artery aneurysmal disease. Catheter Cardiovasc Interv 2008;71:708-14.

14. Behr-Rasmussen C, Grondal N, Bramsen MB, Thomsen MD, Lindholt JS. Mural thrombus and the progression of abdominal aortic aneurysms: a large population-based prospective cohort study. Eur J Vasc Endovasc Surg 2014;48:301-7.

15. Parry DJ, Kessel D, Scott DJ. Simplifying the internal iliac artery aneurysm. Ann R Coll Surg Engl 2001;83:302-8.

16. Johnston KW, Rutherford RB, Tilson MD, Shah DM, Hollier L, Stanley JC. Suggested standards for reporting on arterial aneurysms. Subcommittee on Reporting Standards for Arterial Aneurysms, Ad Hoc Committee on Reporting Standards, Society for Vascular Surgery and North American Chapter, International Society for Cardiovascular Surgery. J Vasc Surg 1991;13:452-8.
17. Zhu C, Haraldsson H, Faraji F, Owens C, Gasper W, Ahn S, Liu J, Laub G, Hope MD, Saloner D. Isotropic 3D black blood MRI of abdominal aortic aneurysm wall and intraluminal thrombus. Magn Reson Imaging 2016;34:18-25.

18. Zhu C, Tian B, Leach JR, Liu Q, Lu J, Chen L, Saloner D, Hope MD. Non-contrast 3D black blood MRI for abdominal aortic aneurysm surveillance: comparison with CT angiography. Eur Radiol 2017;27:1787-94.

19. Whitley E, Ball J. Statistics review 4: sample size calculations. Crit Care 2002;6:335-41.

20. Saam T, Kerwin WS, Chu B, Cai J, Kampschulte A, Hatsukami TS, Zhao XQ, Polissar NL, Neradilek B, Yarnykh VL, Flemming K, Huston J 3rd, Insull W Jr, Morrisett JD, Rand SD, DeMarco KJ, Yuan C. Sample size calculation for clinical trials using magnetic resonance imaging for the quantitative assessment of carotid atherosclerosis. J Cardiovasc Magn Reson 2005;7: 799-808.

21. Sacks NP, Huddy SP, Wegner T, Giddings AE. Management of solitary iliac aneurysms. J Cardiovasc Surg (Torino) 1992;33:679-83.

22. Kasirajan V, Hertzer NR, Beven EG, O'Hara PJ, Krajewski LP, Sullivan TM. Management of isolated common iliac artery aneurysms. Cardiovasc Surg 1998;6:171-7.

23. Sahgal A, Veith FJ, Lipsitz E, Ohki T, Suggs WD, Rozenblit AM, Cynamon J, Wain RA. Diameter changes in isolated iliac artery aneurysms 1 to 6 years after endovascular graft repair. J Vasc Surg 2001;33:289-4; discussion 294-5.

24. Wolf YG, Thomas WS, Brennan FJ, Goff WG, Sise MJ, Bernstein EF. Computed tomography scanning findings associated with rapid expansion of abdominal aortic aneurysms. J Vasc Surg 1994;20:529-35; discussion 535-8.

25. Domonkos A, Staffa R, Kubicek L. Effect of intraluminal thrombus on growth rate of abdominal aortic aneurysms. Int Angiol 2019;38:39-45.

26. Haller SJ, Crawford JD, Courchaine KM, Bohannan CJ, Landry GJ, Moneta GL, Azarbal AF, Rugonyi S. Intraluminal thrombus is associated with early rupture of abdominal aortic aneurysm. J Vasc Surg 2018;67:1051-8.e1.

27. Vorp DA, Lee PC, Wang DH, Makaroun MS, Nemoto EM, Ogawa S, Webster MW. Association of intraluminal thrombus in abdominal aortic aneurysm with local hypoxia and wall weakening. J Vasc Surg 2001;34:291-9. 
28. Speelman L, Schurink GW, Bosboom EM, Buth J, Breeuwer M, van de Vosse FN, Jacobs MH. The mechanical role of thrombus on the growth rate of an abdominal aortic aneurysm. J Vasc Surg 2010;51:19-26.

29. Piechota-Polanczyk A, Jozkowicz A, Nowak W, Eilenberg W, Neumayer C, Malinski T, Huk I, Brostjan C. The

Cite this article as: Wang Y, Zhu C, Leach J, Gasper W, Saloner D, Hope M. Growth of common iliac artery aneurysms coexisting with abdominal aortic aneurysms: associated factors and potential role of intraluminal thrombus. Quant Imaging Med Surg 2020;10(3):703-712. doi: 10.21037/qims.2020.02.12
Abdominal Aortic Aneurysm and Intraluminal Thrombus: Current Concepts of Development and Treatment. Front Cardiovasc Med 2015;2:19.

30. Beales L, Wolstenhulme S, Evans JA, West R, Scott DJ. Reproducibility of ultrasound measurement of the abdominal aorta. Br J Surg 2011;98:1517-25. 